CLINICAL STUDY

\title{
Adrenal vein sampling using rapid cortisol assays in primary aldosteronism is useful in centers with low success rates
}

\author{
Matthias J Betz, Christoph Degenhart ${ }^{1}$, Evelyn Fischer, Anna Pallauf, Volker Brand, Ulrich Linsenmaier ${ }^{1}$, \\ Felix Beuschlein, Martin Bidlingmaier and Martin Reincke \\ Department of Medicine, University Hospital Innenstadt, Ludwig Maximilians University, Ziemssenstrasse 1, D-80336 Munich, Germany and \\ ${ }^{1}$ Department of Clinical Radiology, University Hospital Innenstadt, Ludwig Maximilians University, Nussbaumstrasse 20, D-80336 Munich, Germany \\ (Correspondence should be addressed to M Reincke; Email: martin.reincke@med.uni-muenchen.de)
}

(M J Betz, C Degenhart, M Bidlingmaier and M Reincke contributed equally to this work)

\begin{abstract}
Objective: Adrenal vein sampling (AVS) is considered the gold standard in the differential diagnosis of primary aldosteronism (PA), but success rates vary between centers. We hypothesized that rapid (intraprocedure) cortisol measurement can improve performance in a center with initially low AVS success rate. Design: We analyzed 46 patients with confirmed PA studied between 2008 and 2010. Forty-seven PA patients studied between 2004 and 2008 identified by retrospective chart review served as controls. All patients were treated at a single tertiary care university hospital.

Methods: Starting in 2008, rapid cortisol assays (RCA) were performed in all patients during the AVS procedure. A cortisol gradient of $\geq 2.0$ between adrenal vein and a femoral vein sample was used as success criterion. Up to two repeat samples were drawn if adrenal vein cortisol was below this threshold. Results: During the control period 26 of 47 AVS were successful (55\%). After introduction of RCA, 39 out of 46 AVS $(85 \%)$ were successful $(P=0.003)$. In 21 of the 46 cases $(46 \%)$ a resampling was necessary. The increase in overall success was due to an increase in successful right AVS (85 vs $62 \%$ before introduction of RCA; $P=0.02)$ and a training effect $(P=0.024$ for trend).

Conclusion: RCA during AVS are useful in centers with an initially low AVS success rate.
\end{abstract}

European Journal of Endocrinology 165 301-306

\section{Introduction}

Primary aldosteronism (PA) is a disorder defined by inappropriately high aldosterone secretion in the presence of low to suppressed renin activity and aldosterone levels not suppressible by various suppression tests. While this condition was previously thought to be present in $<1 \%$ of all hypertensive patients, it has lately emerged as one of the most common causes of secondary hypertension, mainly due to the increased use of aldosterone to renin ratio (ARR) as screening test in recent years (1). High aldosterone levels lead to increased potassium excretion, hypokalemia and hypertension. The long-term consequences include myocardial hypertrophy, atrial fibrillation, myocardial infarction, and stroke. Patients with PA suffer from higher cardiovascular and renal morbidity compared with matched patients with essential hypertension (2-4).

PA can be caused by an aldosterone producing adenoma (APA), uni- or bi-lateral adrenal hyperplasia, or in rare cases glucocorticoid remediable aldosteronism. The differential diagnosis of the subtype of PA guides individual medical management. While unilateral disease can be cured by laparoscopic removal of the affected adrenal, mineralocorticoid receptor antagonists are used in bilateral idiopathic hyperaldosteronism to specifically treat the consequences of aldosterone excess.

According to the 2008 Endocrine Society Clinical Practice Guidelines for PA all patients with biochemically confirmed PA should undergo computed tomography (CT) of the adrenal region, to rule out malignancy (2). Due to the often small size of APAs and the high prevalence of non-functioning adrenal adenomas (incidentalomas), adrenal CT as well as magnetic resonance imaging (MRI) both have a relatively low sensitivity and specificity for detecting APAs (5). Therefore, the Endocrine Society recommends establishing subtype diagnosis on the basis of adrenal vein sampling (AVS) in all patients with confirmed PA, who are able and willing to undergo surgery.

However, AVS is a technically challenging and not universally standardized interventional procedure: published success rates for bilateral catheterization vary largely, ranging from 42 to $98 \%$ (6). A retrospective analysis of the data in the German Conn Registry showed an average success rate of only $31 \%$ even at national referral centers (7). 
The adrenal veins are small, feature large anatomical variations and are therefore not easy to catheterize. Particularly, the right adrenal vein often enters the inferior vena cava (IVC) in a steep angle impeding cannulation. Furthermore, adrenal blood flow is estimated to be $\sim 0.2 \%$ of cardiac output (8), which leads to a considerable admixture of blood drawn back from the IVC or the renal vein as well as admixture of blood from accessory hepatic or subphrenic veins. Thus, aldosterone concentrations may vary considerably depending on the exact position of the catheter tip in relation to the orifice of the adrenal vein (9). To account for these variations the aldosterone values are generally corrected by dividing it with the cortisol values measured in the same sample (cortisol-corrected aldosterone ratio). Moreover, the ratio between the cortisol value from the presumed adrenal vein and a sample simultaneously drawn from the IVC or a peripheral vein is used as 'selectivity index' to verify the correct position of the catheter tip. However, the result of the cortisol measurement and therefore confirmation of technical success or failure of the AVS study are often not available until hours or even days after the diagnostic procedure. The patient will already have left the hospital at this time-point, making resampling both cumbersome and expensive.

In 2007, a first proof of concept study was published by an Italian group showing the feasibility of rapid on-site measurement of cortisol during the AVS study in five subjects (10). A second study by Auchus et al. (11) in 30 patients reported that the use of rapid cortisol assays following AVS allowed for resampling on the same day and increased success rate in their hospital.

In this study, we analyzed the effect of RCA on the outcome of AVS in our center that had a low success rate in a historic control group. Data from 46 de novo patients were compared with data from 47 patients, which had previously undergone AVS without RCA.

\section{Subjects and methods}

A systematic retrospective review of the in-house procedures for the diagnosis and differential diagnosis of PA performed within the context of the German Conn's registry (www.conn-registry.de) revealed a low overall success rate of AVS of $55 \%$. To improve the success rates of AVS at our institution, several quality measures were put into place and summarized in a standard operating procedure that was used for all AVS since March 1st 2008.

In detail, the following changes were introduced:

1. As before, a pre-procedural multidetector CT (MDCT) scan was performed to rule out malignancy, according to the Endocrine Society guidelines. In addition, in doing so the late venous scan was used for mapping of the venous anatomy to facilitate super-selective catheterization.
2. AVS were drawn and immediately analyzed for cortisol levels to confirm the correct placement of the catheter. In case of an unsuccessful sampling up to two repeat samplings were performed for each side.

\section{CT technique}

A pre-interventional MDCT scan of the adrenals was performed (late venous phase, $90 \mathrm{~s}$ contrast material delay, $2 \mathrm{ml} / \mathrm{kg}$ body weight) for mapping of the adrenal veins and diagnostic work up. In addition to axial ( $3 \mathrm{~mm}$ slice thickness), multiplanar sagittal and coronal reformations were used ( $3 \mathrm{~mm}$ slice thickness). If no prior imaging study was available, a native CT scan was included.

\section{Adrenal vein sampling}

AVS was performed by three radiologists without concomitant infusion of ACTH 1-24 (Synacthen, Sigma-tau GmbH, Düsseldorf, Germany). The procedure was started at $0800 \mathrm{~h}$. A 5F sheath was introduced via the right common femoral vein; hydrophilic angiography catheters were used to selectively catheterize the adrenal veins. Catheter selection was based on the individual anatomic conditions and cobra, bentson, vertebralis, and sidewinder shape were used respectively. Positioning of the catheter was assessed by venous angiograms before and after selective blood withdrawal. AVS were taken sequentially. A simultaneous sample from the sheath was collected with each of the selective AVS. Finally, samples from the infra- and suprarenal section of IVC were taken, likewise in pair with a sample from the sheath.

Clotting of blood was prevented by collection in tubes prefilled with EDTA (Sarstedt Monovette, Sarstedt, Nümbrecht, Germany). The test tubes were immediately transferred to the endocrinology lab, centrifuged for $5 \mathrm{~min}$ at $3000 \mathrm{~g}$ and the supernatant plasma used for immediate analysis of cortisol. The time for transport, centrifugation and measurement was 25-30 min during which the radiologist attempted sampling of the contralateral adrenal vein. The sampling catheter was not left in position in the adrenal vein during the measurement. The patient stayed in the radiology suite until the last sample was analyzed.

\section{Hormone assays}

Cortisol concentrations were determined using an automated chemiluminescence assay (Liaison, Diasorin, Italy). Aldosterone concentrations were measured using a commercial RIA (Coat-a-count, Siemens, Los Angeles, CA, USA). In our hands, the respective within- and between-assay coefficients of variation were below 
5 and $7 \%$ for cortisol and below 9 and $12 \%$ for aldosterone. All aldosterone samples from each AVS were analyzed in one run. Cortisol was reported in $\mu \mathrm{g} / \mathrm{dl}$ and aldosterone in $\mathrm{pg} / \mathrm{ml}$.

\section{Patients and controls}

Approval from the ethics review board of the LMU Munich was obtained for this chart review study. Patient data for both cohorts was extracted from the German Conn Registry (12) that collected retrospective data up to 2008 and prospective data thereafter. Patients provided formal written informed consent for taking part in the prospective German Conn Registry. Data from 46 de novo patients with PA that underwent AVS starting from March 2008 to October 2010 are analyzed. All patients had arterial hypertension, an elevated ARR and an abnormal suppression test by 2 l of saline infused over $4 \mathrm{~h}$. To study the improvement in successful AVS, we used a historic control cohort of all patients with PA undergoing AVS at our institution between January 2004 and February 2008 before introduction of RCA. Forty-seven patients were identified and AVS success rate was $55 \%$ in the historic cohort (Table 1 ).

\section{Selectivity and lateralization criteria}

Selective adrenal sampling was assumed, if the cortisol concentration of the adrenal vein was at least two times higher than that in the simultaneously drawn sample from the catheter sheath in the right femoral vein (cortisol in adrenal vein/cortisol in right femoral vein $\geq 2.0$ ) (13). The diagnosis of unilateral aldosterone secretion was made, if the aldosterone to cortisol ratio (A/C-ratio) from one adrenal vein was $\geq 4.0$ times the A/C-ratio of the contralateral side (14).

\section{Statistical analysis}

For power calculation, we assumed that the introduction of RCA would improve successful sampling by $50 \%$ over baseline $(=55 \%$ in the historic cohort) to $82 \%$. Following this assumption, we calculated that a minimal sample size of at least 41 for each group would be necessary to demonstrate the superiority of RCA with a statistical power of $80 \%$ and $\alpha<0.05$. For both cohorts, we calculated the overall rate of successful cannulation and success of right and left AVS separately. Continuous data are given as means \pm s.D.

Statistical calculations were performed by Prism Version 5.0 (GraphPad Software, San Diego, CA, USA) by a two-sided Fisher's exact test; $P<0.05$ was considered statistically significant. Logistic regression was performed with IBM SPSS Version 19 (IBM, Armonk, NY, USA).

\section{Results}

\section{Patient characteristics}

Age and sex distribution did not differ significantly between the control and the study period. However, the mean systolic and diastolic blood pressure was higher during the control period $(163 \pm 26$ vs 151 $\pm 22 \mathrm{mmHg}, P=0.04$ and $99 \pm 15$ vs $92 \pm 13 \mathrm{mmHg}$, $P=0.05$ respectively). Plasma potassium, renin, and aldosterone levels did not differ significantly (Table 1).

\section{The percentage of successful AVS increased significantly after the introduction of RCA}

During the control period 26 out of 47 AVS (55\%) resulted in a successful bilateral sampling. After introduction of RCA and immediate resampling the success rate increased to 39 out of $46(84 \%), P=0.003$ (Fig. 1A).

\section{Increased success rate is attributable to the possibility to resample}

To test the possibility that the rate of successful AVS had increased because of factors other than the rapid cortisol assay, we analyzed the rate of successful

Table 1 Patient characteristics of control and of study population. Shown is mean \pm S.D. Differences of the means were analyzed by Student's $t$-test; plasma renin concentrations and aldosterone to renin ratio were log-transformed prior to testing. To convert into SI-units use the following conversion factor: aldosterone, $\mathrm{pg} / \mathrm{ml}$ to $\mathrm{nmol} / \mathrm{l}$, multiply with 0.0277 .

\begin{tabular}{llll}
\hline & Control period & Study period & $P$ \\
\hline$n$ & 47 & 46 & \\
Age (years) & $54 \pm 12$ & $52 \pm 11$ & 0.35 \\
Sex (male/female) & $30 / 17(64 \% / 36 \%)$ & $30 / 16(65 \% / 35 \%)$ & 0.04 \\
Systolic BP (mmHg) & $163 \pm 26$ & $151 \pm 22$ & 0.05 \\
Diastolic BP (mmHg) & $99 \pm 15$ & $92 \pm 13$ & 0.1 \\
Plasma potassium (mmol/l) & $3.4 \pm 0.5$ & $3.5 \pm 0.5$ & 0.1 \\
$\begin{array}{l}\text { Plasma renin concentration (mU/l) } \\
\quad \text { (reference range 2.8-39.9 (supine)) }\end{array}$ & $4.0 \pm 6.5$ & $5.6 \pm 5.7$ & 0.9 \\
$\begin{array}{l}\text { Aldosterone (pg/ml) } \\
\quad \text { (reference range 10-160 (supine)) }\end{array}$ & $295 \pm 227$ & $302 \pm 198$ & 0.2 \\
$\begin{array}{c}\text { Aldosterone/renin - ratio } \\
\quad \text { (reference range <20) }\end{array}$ & $185 \pm 112$ & $129 \pm 190$ & \\
\hline
\end{tabular}




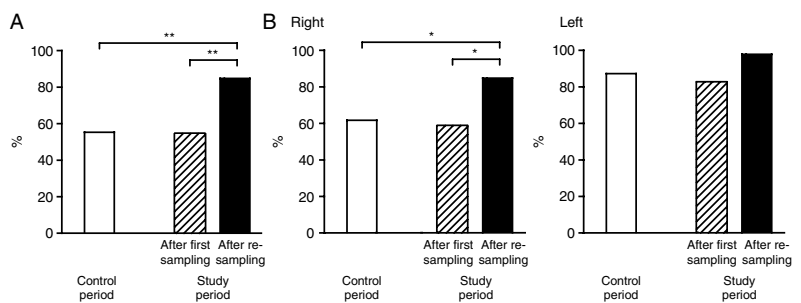

Figure 1 Success rate (in \% of total) of AVS before (control period) and after introduction of RCA (study period). For the study period data are split into the sampling success of the first sampling attempt and the success rate after resampling. (Panel A) Combined success rate of right and left AVS. (Panel B) Success rates divided into right and left AVS. ${ }^{*} P<0.05 ;{ }^{* \star} P<0.01$

sampling at first attempt, i.e. the procedures in which resampling was not necessary and which would have been successful also without the knowledge of the RCA test result. The success rate at first attempt was $54 \%$ and thus not different from the control period $(55 \%$, $P=1.00$ ). On the contrary, the overall success rate after introduction of RCA was significantly higher than success at first sampling attempt ( 85 vs $54 \%, P=0.003$ ).

\section{Increased success is due to resampling of missed right-sided adrenal vein}

The right adrenal vein is generally more difficult to catheterize as it directly enters the IVC. Notably, successful sampling of the right adrenal vein improved from $62 \%$ during the control period to $85 \%$ after introduction of RCA $(P=0.02)$. Similarly, the success rate of sampling from the left adrenal vein increased from $87 \%$ during the control period to $98 \%$ after introduction of RCA albeit this increase failed to reach statistical significance $(P=0.11)$. On the contrary, no improvement was observed for successful samplings at first attempt after introduction of RCA: right adrenal vein 59 vs $62 \%(P=0.834)$ during the control period; left adrenal vein 84 vs $87 \%(P=0.575)$ (Fig. 1B).

\section{Success rate increased over time, while need for resampling decreased}

The overall success rate increased over the time of the study with $75 \%$ for the first 12 diagnostic studies performed during the study period to $100 \%$ for the last 12 AVS ( $P$ for trend $=0.201$ ). In parallel, the need for resampling decreased from $67 \%$ for the first 12 patients to $25 \%$ for the last 12 patients ( $P$ for trend $=0.024$ ), suggesting an effect of radiologist performance (Fig. 2).

\section{Distribution of Iateralization}

Of the 39 successful diagnostic studies, 12 patients were diagnosed having bilateral aldosterone hypersecretion, 13 patients exhibited a lateralization of aldosterone production to the left and 14 to the right (Table 2). The 27 patients with lateralized aldosterone secretion were recommended to undergo minimally invasive adrenalectomy.

\section{Complications}

Hematoma at the site of vascular access was noted in two out of 47 patients during the control period and in two out of 46 patients during the study period. In one patient a peri-adrenal hematoma developed due to adrenal vein perforation during the study period. The hematoma was managed conservatively and the patient left the hospital on the next day without further sequele.

\section{Discussion}

AVS is a key diagnostic procedure in the work up of patients with PA guiding the further management of the patient. However, the procedure is challenging to perform and success rates are often low. Within this study, we could demonstrate that the introduction of rapid cortisol assays significantly improved the success rate of AVS at our institution. While this part of our study is endorsing data reported by Auchus et al. (11) at UTSouthwestern the higher number of patients in our study allows us to analyze the factors contributing to improved success. Specifically, we show that the two major effects of RCA on outcome are due to an initial effect by resampling of the right adrenal vein and a more delayed training effect.

In our study, the success rate previous to the introduction of RCA was unacceptably low compared with centers of excellence. It was considerably lower than at UTSouthwestern ( 55 vs $73 \%$ ) and improved to $85 \%$ (39 of 46 patients) in our cohort in comparison to $97 \%$ at UTSouthwestern (29 of 30 patients). The significant increase in success rate in our series was

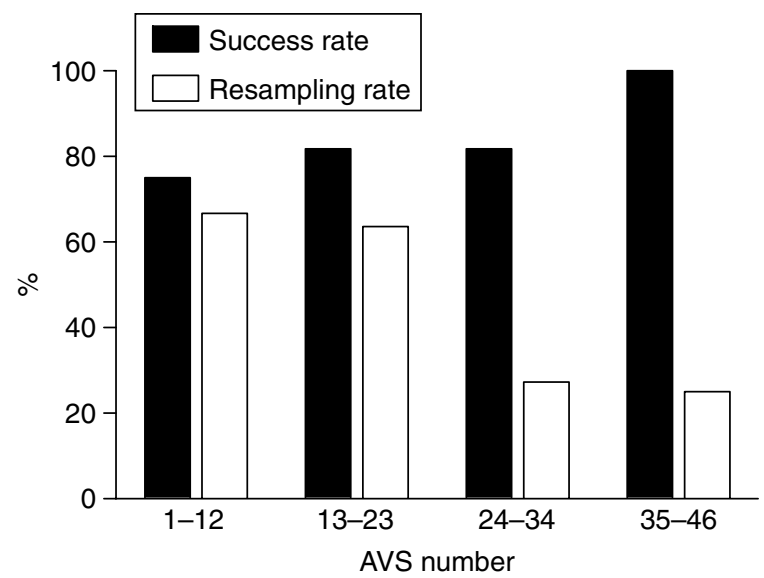

Figure 2 Success rate (black bars) and resampling rate (white bars) as a function of AVS study number. 
Table 2 Results of successful adrenal vein sampling (AVS) during control and study period.

\begin{tabular}{llllllll}
\hline & \multicolumn{3}{c}{ Control period } & & \multicolumn{3}{c}{ Study period } \\
\cline { 2 - 3 } Lateralization & RAV & LAV & Bilateral & & RAV & LAV & Bilateral \\
\hline$n$ & 2 & 10 & 14 & 14 & 13 & 12 \\
$\begin{array}{l}\text { Gradient dominant/ } \\
\text { non-dominant side }\end{array}$ & $86(14)$ & $7(9)$ & $2(2)$ & & $24(25)$ & $13(18)$ & $2(1)$ \\
\hline
\end{tabular}

RAV, right adrenal vein; LAV, left adrenal vein; A/C, aldosterone to cortisol ratio; median (inter-quartile-range).

clearly attributable to the use of RCA. In the Auchus study, the success rate of the first sampling attempt in the studies performed after introduction of RCA did not differ significantly from that of the control period probably because of the already high success rate at baseline before introduction of RCA. Therefore, we believe that RCA is especially useful for centers such as ours with low outcome of AVS at baseline.

One main advantage of the RCA technique implemented at our institution is the use of existing facilities and equipment, limiting investment costs. Due to the close location of the lab to the radiology suite and by using plasma instead of serum thereby eliminating clotting time, we were nonetheless able to achieve a time to results of $25-30 \mathrm{~min}$. The radiologist could, therefore continue the sampling on the contralateral side and the patient stayed in the radiology suite until the examination was completed. However, it must be admitted that this may be quite a unique situation and that other institutions might rather follow the approach used by Mengozzi et al. (10), who used dedicated equipment within the radiology suite, or transfer the patient to the recovery room with the catheter sheath still in place in case of longer times to result (11).

The main limitation of our study is the temporal sequence of the two study periods and the lack of randomization. This study was intended to assess the effect of the implemented measures on AVS success rate, which had been based on earlier studies and guideline recommendations $(2,10)$. Thus, not only the implementation of RCA but also the use of MDCT with mapping of the adrenal veins might have contributed to the improvement. However, the success rate of the first sampling attempt after introduction of RCA did not differ significantly from the control period without RCA guided resampling (Fig. 1). The increase in successfully performed AVS studies was initially attributable to the information obtained through RCA, especially for the right adrenal vein. However, after the first 23 AVS a significant trend toward fewer resampling occurred and persisted until the end of the study (Fig. 2). This is very likely attributable to a training effect, that improved the performance of the interventional radiologists, who indeed judged the rapid feedback through RCA to be very helpful.

The formal success of an AVS study depends largely on the cut off chosen for the selectivity index (15). We used a selectivity index, i.e. the ratio of cortisol in the sample from the adrenal vein to that of a sample simultaneously drawn from the catheter sheath in the femoral vein, of $\geq 2.0$ as the cut off for successful catheterization of the adrenal vein. Other groups have used different values ranging from 1.1 to 5.0 (for review see (5)), the higher cut off values mostly used with concomitant infusion of ACTH 1-24. A recent analysis using different selectivity criteria on a set of patients who underwent AVS twice because of an unsuccessful first AVS, could demonstrate that the technical success of the AVS is dependent on the stringency of the selectivity criteria and that too permissive criteria for successful catheterization lead to poor diagnostic reproducibility (16).

Many groups advocate the simultaneous infusion of ACTH 1-24 during the examination to minimize stress related fluctuations of cortisol and aldosterone. However, there is no consensus on whether or not to use ACTH 1-24 or on the exact modus of administration (bolus versus continuous infusion). One of the advantages of ACTH 1-24 infusion may be a higher step up between peripheral and adrenal vein cortisol values and thus a higher percentage of successful studies. However, the effects on lateralization and thus on diagnosis are not that clear-cut: in a relatively small study with ten patients undergoing AVS first without and then with ACTH 1-24 infusion the authors noted a lateralization toward the wrong side for the values sampled at baseline, suggesting that concomitant ACTH 1-24 infusion might increase the diagnostic reliability (17). A larger study comparing baseline with bolus and continuous infusion of ACTH 1-24 found a completely different effect, claiming that ACTH 1-24 infusion improved the percentage of successful samplings but reduced the number of correctly diagnosed APAs (18). However, these studies used selectivity indices below 2.0 in the baseline setting, which might explain the conflicting results. The role of ACTH 1-24 infusion during AVS remains up to now undetermined.

Despite its lack of standardization and difficulty AVS remains the method of choice for subtype diagnosis of PA, as CT and MRI imaging cannot differentiate between aldosterone producing and inactive adrenal nodules and might not detect small APAs (5). Until newer and more sensitive functional imaging methods overcome these limitations, the success rate of AVS can be reliably increased by rapid cortisol assays, especially in centers with a low initial success rate. 


\section{Declaration of interest}

The authors declare that there is no conflict of interest that could be perceived as prejudicing the impartiality of the research reported.

\section{Funding}

M J Betz is supported by a postdoctoral fellowship from the FritzThyssen-Stiftung and M Reincke by the Deutsche Forschungsgemeinschaft (Re 752/17-1). This study is part of the German Conn's registry-German Else Kröner-Fresenius Registry funded by a grant of the Else Kröner-Fresenius-Stiftung.

\section{Acknowledgements}

We gratefully acknowledge the support of the whole team of the Endocrine Laboratory.

\section{References}

1 Rossi GP, Bernini G, Caliumi C, Desideri G, Fabris B, Ferri C, Ganzaroli C, Giacchetti G, Letizia C, Maccario M, Mallamaci F, Mannelli M, Mattarello MJ, Moretti A, Palumbo G, Parenti G, Porteri E, Semplicini A, Rizzoni D, Rossi E, Boscaro M, Pessina AC \& Mantero F. A prospective study of the prevalence of primary aldosteronism in 1,125 hypertensive patients. Journal of the American College of Cardiology 200648 2293-2300. (doi:10. 1016/j.jacc.2006.07.059)

2 Funder JW, Carey RM, Fardella C, Gomez-Sanchez CE, Mantero F, Stowasser M, Young WF Jr \& Montori VM. Case detection, diagnosis, and treatment of patients with primary aldosteronism: an endocrine society clinical practice guideline. Journal of Clinical Endocrinology and Metabolism 200893 3266-3281. (doi:10. 1210/jc.2008-0104)

3 Matsumura K, Fujii K, Oniki H, Oka M \& Iida M. Role of aldosterone in left ventricular hypertrophy in hypertension. American Journal of Hypertension 200619 13-18. (doi:10.1016/ j.amjhyper.2005.05.013)

4 Rossi GP, Bernini G, Desideri G, Fabris B, Ferri C, Giacchetti G, Letizia C, Maccario M, Mannelli M, Matterello MJ, Montemurro D, Palumbo G, Rizzoni D, Rossi E, Pessina AC \& Mantero F. Renal damage in primary aldosteronism: results of the PAPY study. Hypertension $2006 \mathbf{4 8}$ 232-238. (doi:10.1161/01.HYP. $0000230444.01215 .6 a)$

5 Kempers MJ, Lenders JW, van Outheusden L, van der Wilt GJ, Schultze Kool LJ, Hermus AR \& Deinum J. Systematic review: diagnostic procedures to differentiate unilateral from bilateral adrenal abnormality in primary aldosteronism. Annals of Internal Medicine 2009151 329-337.

6 Stewart PM \& Allolio B. Adrenal vein sampling for primary aldosteronism: time for a reality check. Clinical Endocrinology 2010 72 146-148. (doi:10.1111/j.1365-2265.2009.03714.x)

7 Vonend O, Ockenfels N, Gao X, Allolio B, Lang K, Mai K, Quack I, Saleh A, Degenhart C, Seufert J, Seiler L, Beuschlein F, Quinkler M, Podrabsky P, Bidlingmaier M, Lorenz R, Reincke M \& Rump LC. Adrenal venous sampling: evaluation of the German Conn's Registry. Hypertension 201157 990-995. (doi:10.1161/HYPERTENSIONAHA.110.168484)

8 Williams LR \& Leggett RW. Reference values for resting blood flow to organs of man. Clinical Physics and Physiological Measurement 198910 187-217. (doi:10.1088/0143-0815/10/3/001)
9 Auchus RJ, Wians FH Jr, Anderson ME, Dolmatch BL, Trimmer CK, Josephs SC, Chan D, Toomay S \& Nwariaku FE. What we still do not know about adrenal vein sampling for primary aldosteronism. Hormone and Metabolic Research 201042 411-415. (doi:10.1055/ s-0030-1252060)

10 Mengozzi G, Rossato D, Bertello C, Garrone C, Milan A, Pagni R, Veglio F \& Mulatero P. Rapid cortisol assay during adrenal vein sampling in patients with primary aldosteronism. Clinical Chemistry 200753 1968-1971. (doi:10.1373/clinchem.2007. 092080)

11 Auchus RJ, Michaelis C, Wians FH Jr, Dolmatch BL, Josephs SC, Trimmer CK, Anderson ME \& Nwariaku FE. Rapid cortisol assays improve the success rate of adrenal vein sampling for primary aldosteronism. Annals of Surgery 2009249 318-321. (doi:10. 1097/SLA.0b013e3181961d77)

12 Schirpenbach C, Segmiller F, Diederich S, Hahner S, Lorenz R, Rump LC, Seufert J, Quinkler M, Bidlingmaier M, Beuschlein F, Endres S \& Reincke M. The diagnosis and treatment of primary hyperaldosteronism in Germany: results on 555 patients from the German Conn Registry. Deutsches Ärzteblatt International 2009 106 305-311.

13 Mulatero P, Bertello C, Rossato D, Mengozzi G, Milan A, Garrone C, Giraudo G, Passarino G, Garabello D, Verhovez A, Rabbia F \& Veglio F. Roles of clinical criteria, computed tomography scan, and adrenal vein sampling in differential diagnosis of primary aldosteronism subtypes. Journal of Clinical Endocrinology and Metabolism 200893 1366-1371. (doi:10.1210/jc.2007-2055)

14 Mathur A, Kemp CD, Dutta U, Baid S, Ayala A, Chang RE, Steinberg SM, Papademetriou V, Lange E, Libutti SK, Pingpank JF, Alexander HR, Phan GQ, Hughes M, Linehan WM, Pinto PA, Stratakis CA \& Kebebew E. Consequences of adrenal venous sampling in primary hyperaldosteronism and predictors of unilateral adrenal disease. Journal of the American College of Surgeons 2010211 384-390. (doi:10.1016/j.jamcollsurg.2010. 05.006)

15 Rossi GP, Pitter G, Bernante P, Motta R, Feltrin G \& Miotto D. Adrenal vein sampling for primary aldosteronism: the assessment of selectivity and lateralization of aldosterone excess baseline and after adrenocorticotropic hormone (ACTH) stimulation. Journal of Hypertension 200826 989-997. (doi:10.1097/HJH. Ob013e3282f9e66a)

16 Mulatero P, Bertello C, Sukor N, Gordon R, Rossato D, Daunt N, Leggett D, Mengozzi G, Veglio F \& Stowasser M. Impact of different diagnostic criteria during adrenal vein sampling on reproducibility of subtype diagnosis in patients with primary aldosteronism. Hypertension 201055 667-673. (doi:10.1161/HYPERTENSIONAHA.109.146613)

17 Tanemoto M, Suzuki T, Abe M, Abe T \& Ito S. Physiologic variance of corticotropin affects diagnosis in adrenal vein sampling. European Journal of Endocrinology 2009160 459-463. (doi:10. 1530/EJE-08-0840)

18 Seccia TM, Miotto D, De Toni R, Pitter G, Mantero F, Pessina AC \& Rossi GP. Adrenocorticotropic hormone stimulation during adrenal vein sampling for identifying surgically curable subtypes of primary aldosteronism: comparison of 3 different protocols. Hypertension 200953 761-766. (doi:10.1161/HYPERTENSIONAHA.108.128553)

Received 13 May 2011

Accepted 19 May 2011 